This article is licensed under the Creative Commons Attribution-NonCommercial 4.0 International License (CC BY-NC) (http://www.karger.com/Services/OpenAccessLicense). Usage and distribution for commercial purposes requires written permission.

\title{
Progression from an Inflammatory to a Fibrostenotic Phenotype in Eosinophilic Esophagitis
}

\author{
Nathaniel T. Koutlas Evan S. Dellon \\ Center for Esophageal Disease and Swallowing, Division of Gastroenterology and \\ Hepatology, University of North Carolina School of Medicine, Chapel Hill, NC, USA
}

\section{Keywords}

Eosinophilic esophagitis · Progression · Natural history $\cdot$ Stricture $\cdot$ Dilation

\begin{abstract}
Previous studies have suggested that eosinophilic esophagitis (EoE) progresses from chronic inflammation to fibrostenosis. However, natural history data illustrating this progression in individual patients are lacking. Here, we present 6 patients who progressed from an inflammatory to a fibrostenotic phenotype of EoE in the absence of treatment. At the time of diagnosis, none of the patients had significant evidence of fibrostenosis, but they did have other inflammatory findings of EoE such as edema, linear furrows, or exudates. After being lost to follow-up and treatment for an average of $7.8 \pm 2.0$ years, strictures $(n=5 ; 83 \%)$ or a smallcaliber esophagus $(n=4 ; 67 \%)$ were present in a majority of the patients, and the majority required esophageal dilation. These cases confirm that EoE can progress from an inflammation-only phenotype to a fibrostenotic phenotype in certain patients.
\end{abstract}




\section{Case Reports in Gastroenterology}

\section{Introduction}

Eosinophilic esophagitis (EoE) is a chronic immune/allergen-mediated disorder of the esophagus characterized by symptoms of esophageal dysfunction and eosinophilia on esophageal biopsy $[1,2]$. It has been suggested that chronic eosinophilic inflammation (often manifest on endoscopy with white exudates, edema, and linear furrows) progresses to fibrostenosis (with esophageal rings, strictures, or narrowing) if it persists over time [3-6]. However, these claims are largely based on cross-sectional data, and natural history data are limited. The aim of this study is to present a series of patients who progressed from inflammatory to fibrostenotic phenotypes of EoE in the absence of treatment.

\section{Case Reports}

\section{Case 1}

A 14-year-old male with asthma was diagnosed with EoE in July 2004 with several years of symptoms of solid food dysphagia and vomiting. He presented with a food impaction that required urgent endoscopic removal of the food bolus. On the initial upper endoscopy (Fig. $1 \mathrm{a})$, there was edema, furrows, and friability but no evidence of strictures or narrowing. He was lost to follow-up for personal reasons until March 2013. At this time, he presented with worsening solid food dysphagia after every meal, regurgitation of the stuck food, and pill dysphagia, as well as transient food impactions. Follow-up endoscopy, which required use of a neonatal upper endoscope to traverse the esophagus, showed rings, edema, furrows, exudates, a small-caliber esophagus, and a focal stricture with an inner diameter estimated at 10-11 mm (Fig. 1b). Esophageal dilation (Savary) was performed, with a dilation effect at $11 \mathrm{~mm}$.

Case 2

An 18-year-old male with eczema and seasonal allergies initially presented with a 1year history of dysphagia primarily to pills and suspected pill impaction in June 2004. Initial endoscopy showed edema, erythema, and friability. He was lost to follow-up for personal reasons until November 2011, when he presented to the emergency department with a food impaction. He provided an additional history of intermittent solid food dysphagia over the intermediate 7 years. Repeat endoscopy showed rings, furrows, edema, and a small-caliber esophagus with a proximal stricture $8 \mathrm{~mm}$ in diameter. There was mucosa disruption with passage of the endoscope, so dilation was deferred.

Case 3

A 30-year-old male was diagnosed with EoE in November 2008. He presented with a 15year history of intermittent solid food dysphagia. His initial endoscopy had findings of rings, furrows, and a focal area of mild stricture in the proximal esophagus, but an adult upper endoscope could pass without resistance (Fig. 1c). He was lost to follow-up for personal reasons until April 2015, when he presented with worsening solid food and liquid dysphagia, as well as frequent transient food impactions and a 30-lb weight loss secondary to reduced oral intake. A repeat endoscopy was significant for rings, furrows, edema, and a small-caliber 
esophagus, with the focal stricture measuring $3 \mathrm{~mm}$ in diameter, which precluded the passage of a neonatal endoscope (Fig. 1d). Savary dilation was performed up to $5 \mathrm{~mm}$, but the 7 $\mathrm{mm}$ dilator was unable to pass.

\section{Case 4}

A 12-year-old male was diagnosed with EoE in August 2004. He presented with 1 year of intermittent solid food dysphagia and a transient food impaction that did not require urgent endoscopy. His initial endoscopy showed only mild esophagitis. He was lost to follow-up care until November 2013, when he presented with a frank food impaction requiring endoscopic removal of the food bolus. Upper endoscopy at that time showed rings, furrows, and a stricture measuring $<9 \mathrm{~mm}$ in diameter at the gastroesophageal junction. Because of focal trauma at the food bolus impaction site, dilation was deferred.

\section{Case 5}

A 10-year-old male initially presented in June 2006 with a 1-year history of heartburn, intermittent solid food dysphagia, and transient food impactions. Upper endoscopy was significant for mild esophagitis but was otherwise normal. He was lost to follow-up until August 2016 for personal reasons. At that time, he reported solid food dysphagia with every meal and frequent transient food impactions that had progressively worsened over the past year. On repeat endoscopy, a neonatal endoscope was required to traverse the esophagus, and there were exudates, rings, edema, furrows, and a small-caliber esophagus with a focal stricture in the distal esophagus measuring $9 \mathrm{~mm}$. Savary dilation was performed to $11 \mathrm{~mm}$.

\section{Case 6}

A 9-year-old female with a history of multiple food allergies was diagnosed with EoE in 2000. She was then lost to follow-up and re-presented 5 years later with several years of intermittent abdominal pain and heartburn. Her initial endoscopy showed mild esophagitis characterized by edema and furrows (Fig. 1e). She was again inconsistent with follow-up and treatment until October 2011. Her symptoms had progressed to more frequent upper abdominal pain as well as solid food dysphagia. Her endoscopy at that time showed diffuse rings, narrowing, furrows, edema, and exudates, but without a clear focal stricture (Fig. 1f).

\section{Discussion}

EoE is thought to progress from an inflammatory to a fibrostenotic phenotype in some patients. Several studies have noted differences in endoscopic findings and clinical symptoms between children and adults, suggesting progression from an inflammatory to a fibrostenotic phenotype [7-9]. In general, nonspecific symptoms such as failure to thrive, abdominal pain, heartburn, and vomiting are seen more commonly in pediatric patients; adults more frequently report typical symptoms such as food impaction and dysphagia $[7,8]$. On endoscopy, children often have a normal-appearing esophagus or various inflammatory findings, while fibrostenotic findings are more commonly seen in adults $[8,9]$.

Three previous studies examined the risk of developing strictures or fibrostenosis with diagnostic delay in EoE patients [3-5]. However, these studies relied on cross-sectional data. 
To date, there has been little direct natural history evidence on individual patients to support these claims. Our study presents 6 patients initially diagnosed with EoE as children without significant fibrostenosis reported. Each patient was lost to follow-up for an average of $7.8 \pm 2.0$ years and later returned with rings and/or strictures. Additionally, 4 of the patients developed a small-caliber esophagus (Table 1). Natural history data on EoE is difficult to obtain as patients are now treated immediately upon diagnosis. Previous natural history data did not specifically address fibrostenosis [10]. This case series is important as it provides proof of principle of progression in EoE, and to date such a series has not been published.

There are several limitations to our study. First, this is a retrospective study reliant upon previous clinical and endoscopic reports authored by different attending physicians. Thus, there were likely differences in the reporting of endoscopic findings. Second, endoscopic images were not available for all patients, and differences in endoscope resolution may have impacted the reporting of endoscopic findings at baseline. However, we do not feel that prominent rings or strictures, especially those that would preclude endoscope passage, would be missed.

In summary, our study demonstrated the progression of EoE from chronic inflammation to fibrostenosis in a group of 6 patients in the absence of treatment. This study provides proof of the progression of EoE in some patients and highlights the necessity of treatment to prevent fibrostenosis and its associated complications.

\section{Statement of Ethics}

This project was deemed exempt from ongoing review by the UNC IRB. All identifying patient information has been removed to protect patient privacy.

\section{Disclosure Statement}

None of the authors have relevant disclosures related to this paper. This study was funded in part by NIH T35DK007386 (N.T.K.).

\section{Author Contributions}

E.S.D. is the guarantor of the paper. The following are the roles: N.T.K., data collection, manuscript drafting, critical revision, and final approval; E.S.D., project conception, data collection, critical revision, project supervision, and final approval. 
Koutlas and Dellon: Progression from an Inflammatory to a Fibrostenotic Phenotype in Eosinophilic Esophagitis

\section{References}

1 Dellon ES, Gonsalves N, Hirano I, Furuta GT, Liacouras C, Katzka DA: ACG clinical guideline: evidence based approach to the diagnosis and management of esophageal eosinophilia and eosinophilic esophagitis (EoE). Am J Gastroenterol 2013;108:679-692; quiz 693.

$\checkmark 2$ Liacouras CA, Furuta GT, Hirano I, Atkins D, Attwood SE, Bonis PA, et al: Eosinophilic esophagitis: updated consensus recommendations for children and adults. J Allergy Clin Immunol 2011;128: 3-20.e6; quiz 21-22.

-3 Dellon ES, Kim HP, Sperry SL, Rybnicek DA, Woosley JT, Shaheen NJ: A phenotypic analys is shows that eosinophilic esophagitis is a progressive fibrostenotic disease. Gastrointest Endosc 2014;79:577585.e4.

-4 Schoepfer AM, Safroneeva E, Bussmann C, Kuchen T, Portmann S, Simon HU, et al: Delay in diagnosis of eosinophilic esophagitis increases risk for stricture formation in a time-dependent manner. Gastroenterology 2013;145:1230-1236.e1-2.

$\checkmark 5$ Lipka S, Kumar A, Richter JE: Impact of diagnostic delay and other risk factors on eosinophilic esophagitis phenotype and esophageal diameter. J Clin Gastroenterol 2016;50:134-140. Hirano I, Aceves SS: Clinical implications and pathogenesis of esophageal remodeling in eosinophilic esophagitis. Gastroenterol Clin North Am 2014;43:297-316. Noel RJ, Putnam PE, Rothenberg ME: Eosinophilic esophagitis. N Engl J Med 2004;351:940-941. Dellon ES, Gibbs WB, Fritchie KJ, Rubinas TC, Wilson LA, Woosley JT, et al: Clinical, endoscopic, and histologic findings distinguish eosinophilic esophagitis from gastroesophageal reflux disease. Clin Gastroenterol Hepatol 2009;7:1305-1313; quiz 1261. Kim HP, Vance RB, Shaheen NJ, Dellon ES: The prevalence and diagnostic utility of endoscopic features of eosinophilic esophagitis: a meta-analysis. Clin Gastroenterol Hepatol 2012;10:988-996.e5. Straumann A, Spichtin HP, Grize L, Bucher KA, Beglinger C, Simon HU: Natural history of primary eosinophilic esophagitis: a follow-up of 30 adult patients for up to 11.5 years. Gastroenterology 2003;125:1660-1669. 


\section{Case Reports in Gastroenterology}
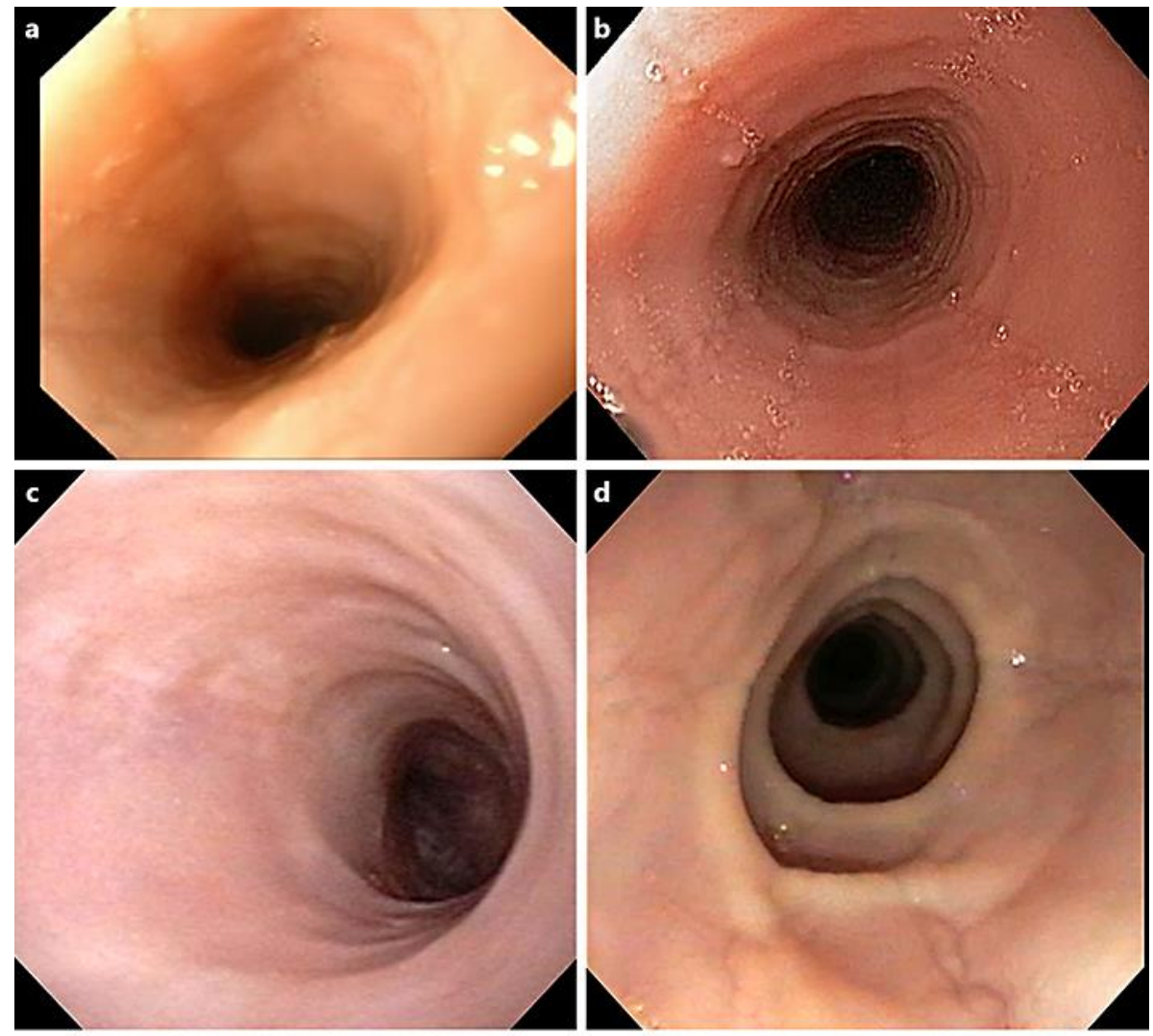

e

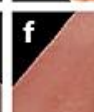

Age:
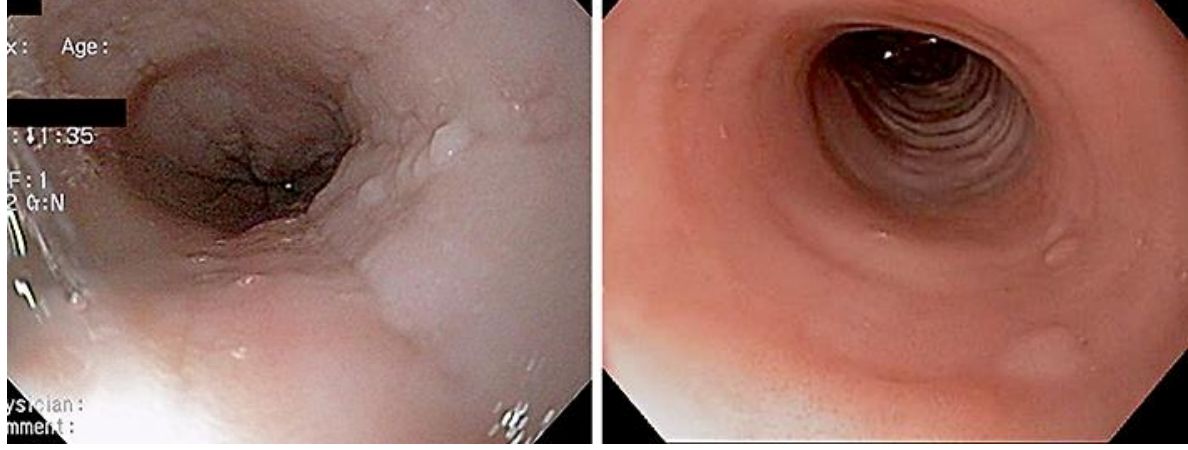

Fig. 1. a Initial endoscopic appearance of case 1, showing edema and furrows but a patent lumen. $\mathbf{b}$ Followup endoscopic appearance of case 1, demonstrating rings and narrowing, as well as furrows, plaques, and edema. c Baseline endoscopic appearance of case 3, showing edema and mild rings. $\mathbf{d}$ Follow-up endoscopy of case 3, showing tight rings and marked narrowing, as well as edema and furrows. e First endoscopic appearance of case 6 , showing edema and furrows, but no narrowing, rings, or stricture. f On follow-up, case 6 now had narrowing and prominent rings, as well as persistent edema. 
Koutlas and Dellon: Progression from an Inflammatory to a Fibrostenotic Phenotype in

Table 1. Patient characteristics

Mean age at diagnosis \pm SD, years

$14.6 \pm 8.3$

Male, $n(\%)$

$5(83)$

Mean time out of care \pm SD, years

$7.8 \pm 2.0$

Baseline endoscopic findings, $n(\%)$

Exudates

$0(0)$

Rings

$2(33)$

Edema

2 (33)

Furrows

2 (33)

Stricture

1 (17)

Small-caliber esophagus

$0(0)$

Crepe-paper mucosa

$0(0)$

Esophagitis

5 (83)

Dilation

1 (17)

Follow-up endoscopic findings, $n(\%)$

Exudates

$3(50)$

Rings

$6(100)$

Edema

5 (83)

Furrows

$6(100)$

Stricture

$5(83)$

Small-caliber

4 (67)

Crepe-paper mucosa

1 (17)

Esophagitis

$0(0)$

Dilation

4 (67)

Mean baseline max. eosinophil count per high-power field \pm SD

$125 \pm 120$

Mean follow-up max. eosinophil count per high-power field \pm SD

$72.3 \pm 45$ 